\title{
Successful treatment of interstitial pneumonia with autoimmune features using rituximab as salvage therapy
}

\author{
Abeline Kapuczinski ${ }^{1}$, Michel Cotils ${ }^{1}$, and Yannick Gombeir ${ }^{1}$ \\ ${ }^{1}$ Hospital Centre EpiCURA Hornu
}

February 27, 2021

\begin{abstract}
Interstitial pneumonia with autoimmune features is a concept to identify patients with idiopathic interstitial pneumonia and features suggestive of a connective tissue disease. There is no specific treatment which consists mainly of corticosteroids and immunossupressive agents. Rituximab is an effective rescue therapy for refractory interstitial lung disease.
\end{abstract}

\section{Introduction}

Some patients with an idiopathic interstitial pneumonia (IIP) have autoimmune features but do not fulfill established criteria for a connective tissue disease (CTD) (1). In 2015, the « European Respiratory Society (ERS) and American Thoracic Society (ATS) Task Force on Undifferentiated Forms of Connective tissue disease-associated interstitial lung disease $\gg$ proposed the term $\ll$ interstitial pneumonia with autoimmune features $\gg($ IPAF) (2). The classification criteria for IPAF is a combination of three domains : clinical features, serologic features and morphologic features. The physiopathology of the disease remains unclear and there is no specific treatment so that corticosteroids and immunosuppressive agents are the mainstay of the treatment (1).

Interstitial lung disease (ILD) is associated with considerable morbidity and mortality. There can be a lack of therapeutic response in very severe lung involvement despite maximal conventional treatment, in such a way that rituximab (Rtx) may be an effective medical rescue therapy for ILD unresponsive to conventional immunosupressive treatment (3).

We present a patient with IPAF treated successfully with rituximab used as salvage therapy.

\section{Case description}

A 49-year-old man came to the Emergency Department after the first wave of Coronavirus because of dyspnea, fever and arthralgia. He is a farmer and his medical history includes type 1 diabetes and asthma. General examination was normal except lung crackles. His temperature was of $38.5^{\circ} \mathrm{C}$ with an oxygen saturation of $95 \%$ to ambient air. Laboratory tests revealed a C-reactive protein level of $31 \mathrm{mg} / \mathrm{l}$ and a natremia of 122 $\mathrm{mmol} / \mathrm{l}$. CK levels were in standards. Computed tomography of the chest showed an interstitial pneumonia suspected of SARS-CoV-2 infection and the patient was hospitalised in the Covid-19 ward of the hospital (Figure 1 ). Later, viral serologies and bacterial cutures were all negative, including screening HIV and hepatitis. An antibiotherapy by amoxicillin/clavulanic acid was started and since the patient needed oxygen supplementation, he was admitted to the Intensive Care Unit (ICU) after a week of hospitalisation. An escalation therapy by piperacillin/tazobactam and doxycycline was done with oxygen therapy of 8 litres per minute by mask but intubation was never required. Bronchoalveolar lavage showed a lot of macrophages and a low CD4/CD8 ratio with bacteriological and Pneumocystis Jiroveci research negatives. Antinuclear antibodies (ANA) were positives with a 1/2640 titre and anti-RNP and anti-JO1 identification. IPAF was 
established and a treatment with corticosteroids and mycophenolate mofetil (MMF) was started in the ICU. The patient was then transferred to the Internal Medecine Department for his management. Unfortunately, his need of oxygen did not decrease so that supplemental oxygen with 8 litres per minute was required for oxygen saturation of $92 \%$ in a resting condition. He desaturated with exercises at $80 \%$ of saturation. Pulmonary function tests were unrealisable at that time. After a month without improvement, the patient received rituximab (Rtx) as salvage therapy and finally got better. After a few days, his need of oxygen decreased and he went to a rehabilitation centre for recovery. At a 4-month follow-up, he no longer needed oxygen and control computed tomography of the chest showed significant improvement (Figure 2 ).

\section{Discussion}

IPAF is not really a diagnosis, but a term used for patients with idiopathic interstitial pneumonia and features of autoimmunity who did not fall within the criteria of a connective tissue disease (4). In 2015, a multidiscipinary task force proposed classification criteria for IPAF. Those criteria are organised around features of three domains : a clinical domain consisting of specific extra-thoracic features, a serologic domain consisting of specific autoantibodies, and a morphologic domain consisting of specific chest imaging, histopathology or pulmonary physiologic features (2). According to Fischer et al., our patient had the criteria with an idiopathic interstitial pneumonia based on the CT scan, ANA > $1: 320$ titre (anti-RNP and anti-JO1 identification) and diffuse polyarthralgia. Furthermore, all alternative etiologies were excluded and he did not meet criteria of a defined connective tissue disease. We also note that CK levels were in standards and the patient did not present myalgia despite positive anti-JO1 identification.

There is no specific treatment as IPAF is a research consensus statement and not a well-defined entity. Management consists of global care with pulmonary rehabilitation, oxygen therapy if necessary, prevention of infection and bone health assessment (1). Corticosteroids and immunosuppressive agents are usually used. For example, azathioprine and mycophenolate mofetil (MMF) are associated with improvement or stabilization of lung function (1). In this case, the patient received a combination of corticosteroids and MMF but this was not enough. He was considered as a patient with refractory interstitial lung disease (ILD) and Rtx treatment was given as salvage therapy with an improved therapeutic response. A retrospective study indicates efficacy of Rtx in patients with Antisynthetase Syndrome (AS) and ILD. Indeed, this study demonstrated a reduction in ILD extent post therapy (5). Doyle et al. also showed that in ILD with AS, the principal indication for Rtx use was recurrent or progressive ILD due to failure of other agents resulting in stability or improvement in pulmonary function or severity of ILD on CT scan with a good tolerance of the treatment (6).

In 2012, Keir et al. showed the benefits of using Rtx as life-saving therapeutic intervention in severe CTDILD with failure to conventional immunosuppression. In his study, patients improved pulmonary function with Rtx (3). In 2016, Lepri et al. demonstrated that Rtx may help to control the course of ILD in CTD producing a stabilisation of lung involvement in systemic sclerosis and mixed connective tissue disorders (7).

According to Saunders et al., there are no approved therapies for CTD-ILD : cyclophosphamide is the main therapy for patient with severe or progressive CTD-ILD but observationnal studies suggested that Rtx is an effective rescue therapy for refractory CTD-ILD (8).

Recently, Atienza-Mateo et al. demonstrated that Rtx was a good therapeutic option to preserve lung fonction in patients with IPAF (9).

\section{Conclusion}

IPAF is a reasearch classification with no specific treatment due to its heterogeneity. Management should be discussed on a case-by-case basis in a pluri-disciplinary way. More studies should be done to achieve an effective therapeutic approach. Rituximab should be considered in patients with severe lung involvement.

\section{Conflicts of Interests}

The Authors declare that there are no competing interests. 


\section{Author contributions}

AK wrote the manuscript. YG et MC were actively involved in the clinical care of the patient. All authors read and approved the final manuscript.

\section{References}

1. Fernandes L, Nasser M, Ahmad K, Cottin V. Interstitial Pneumonia With Autoimmune Features (IPAF). Front Med (Lausanne). 2019;6:209.

2. Fischer A, Antoniou KM, Brown KK, Cadranel J, Corte TJ, du Bois RM, et al. An official European Respiratory Society/American Thoracic Society research statement: interstitial pneumonia with autoimmune features. Eur Respir J. 2015 Oct;46(4):976-87.

3. Keir GJ, Maher TM, Ming D, Abdullah R, de Lauretis A, Wickremasinghe M, et al. Rituximab in severe, treatment-refractory interstitial lung disease. Respirology. 2014 Apr;19(3):353-9.

4. Graney BA, Fischer A. Interstitial Pneumonia with Autoimmune Features. Ann Am Thorac Soc. 2019 May;16(5):525-33.

5. Andersson H, Sem M, Lund MB, Aaløkken TM, Günther A, Walle-Hansen R, et al. Long-term experience with rituximab in anti-synthetase syndrome-related interstitial lung disease. Rheumatology (Oxford). 2015 Aug;54(8):1420-8.

6. Doyle TJ, Dhillon N, Madan R, Cabral F, Fletcher EA, Koontz DC, et al. Rituximab in the Treatment of Interstitial Lung Disease Associated with Antisynthetase Syndrome: A Multicenter Retrospective Case Review. J Rheumatol. 2018 Jun;45(6):841-50.

7. Lepri G, Avouac J, Airò P, Anguita Santos F, Bellando-Randone S, Blagojevic J, et al. Effects of rituximab in connective tissue disorders related interstitial lung disease. Clin Exp Rheumatol. 2016 Oct;34 Suppl 100(5):181-5.

8. Saunders P, Tsipouri V, Keir GJ, Ashby D, Flather MD, Parfrey H, et al. Rituximab versus cyclophosphamide for the treatment of connective tissue disease-associated interstitial lung disease (RECITAL): study protocol for a randomised controlled trial. Trials. 2017 Jun 15;18(1):275.

9. Atienza-Mateo B, Remuzgo-Martínez S, Prieto-Peña D, Mora Cuesta VM, Iturbe-Fernández D, Llorca J, et al. Rituximab in the Treatment of Interstitial Lung Disease Associated with Autoimmune Diseases: Experience from a Single Referral Center and Literature Review. J Clin Med [Internet]. 2020 Sep 23 [cited 2020 Dec 28];9(10). Available from: https://www.ncbi.nlm.nih.gov/pmc/articles/PMC7598697/

Figure 1. Computed tomography of the chest at the admission Figure 2. Computed tomography of the chest at a 4 - mont 

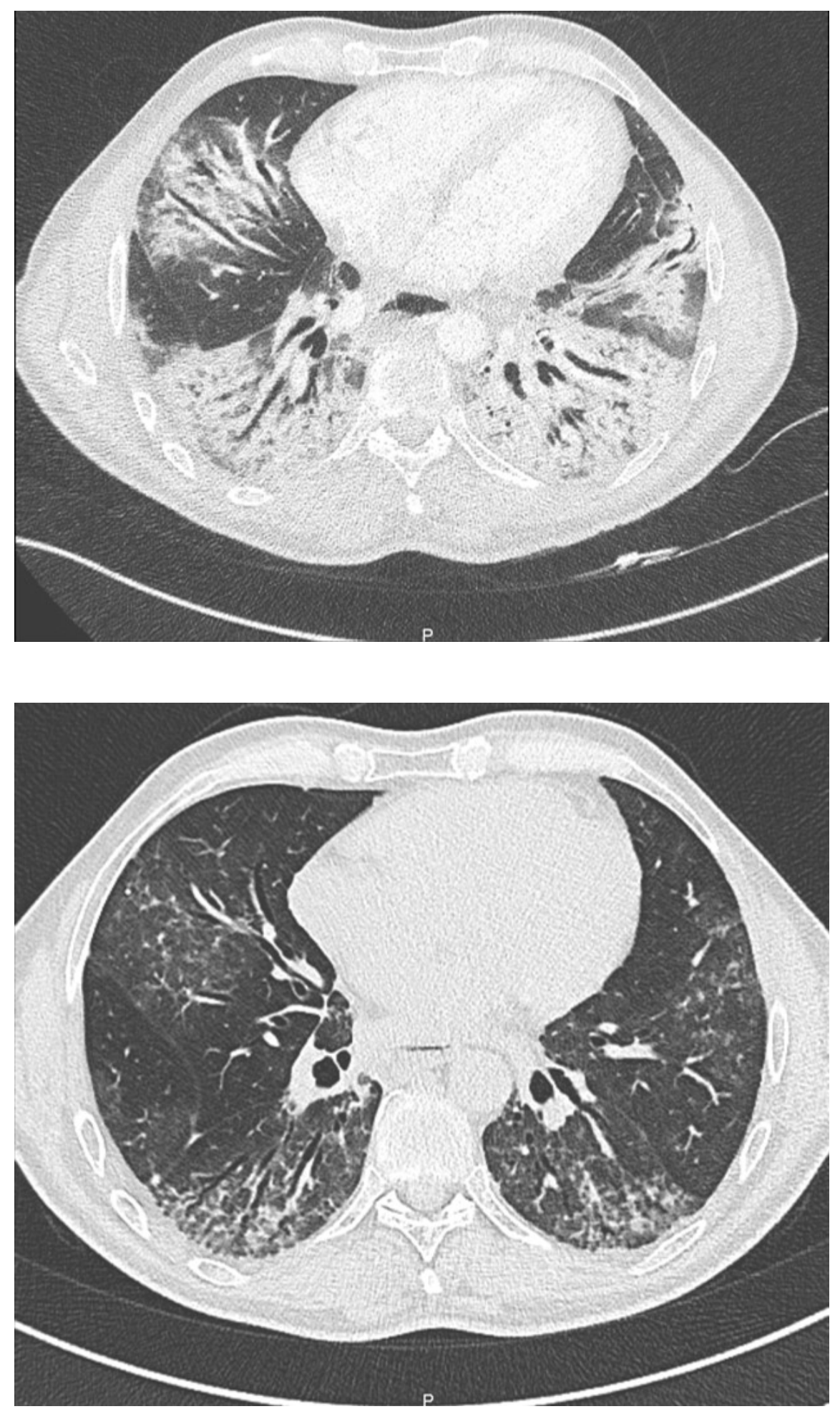